\title{
SHORT REPORT \\ Feasibility of a rubella screening and vaccination programme for unvaccinated young women
}

\author{
W. L. M. RUIJS ${ }^{1,2 *}$, M. E. J. L. HULSCHER ${ }^{3}$, S. J. M. HAHNÉ ${ }^{4}$, \\ R. S. VAN BINNENDIJK ${ }^{4}$ AND J. VAN DER VELDEN ${ }^{1}$ \\ ${ }^{1}$ Radboud University Nijmegen Medical Centre, department of Public Health, Nijmegen, The Netherlands \\ ${ }^{2}$ GGD Rivierenland, Municipal Health Service, Tiel, The Netherlands \\ ${ }^{3}$ Radboud University Nijmegen Medical Centre, Centre for Quality of Care Research, Nijmegen, The Netherlands \\ ${ }^{4}$ National Institute for Public Health and the Environment, Centre for Infectious Disease Control, Bilthoven, \\ The Netherlands
}

(Accepted 15 December 2008; first published online 4 February 2009)

\section{SUMMARY}

The feasibility of a rubella screening and vaccination programme for unvaccinated young women was assessed after the 2004/2005 epidemic in The Netherlands. All 640 young women in two villages with low vaccination coverage were invited for a rubella seroprevalence test. Information on vaccination status was gathered by written questionnaire. Women testing seronegative were offered free rubella vaccination. The feasibility of the programme was evaluated in terms of participation, rubella susceptibility, and acceptance of the vaccination offer by seronegative women. The participation rate was $48 \%$ [95\% confidence interval (CI) 44-52] with 108 unvaccinated participants. Eleven per cent (95\% CI 6-19) of the women were identified as susceptible to rubella, of whom $17 \%$ (95\% CI 2-48) accepted the vaccination offer. In the end only $0.9 \%(95 \%$ CI $0 \cdot 1-2 \cdot 5)$ of the target population was given protection by the programme. Under the present conditions this programme proved to be an inefficient strategy for rubella protection.

Key words: Rubella, screening, vaccination.

Rubella is generally a mild infectious disease. However, during pregnancy it may cause serious congenital malformations in the foetus known as congenital rubella syndrome (CRS). Rubella and CRS can be prevented by vaccination [1] and in The Netherlands rubella vaccination was introduced for girls only as part of a national vaccination programme in 1974. In 1987, this was replaced by the two-dose measles-mumps-rubella (MMR) vaccination for all

\footnotetext{
* Author for correspondence: W. L. M. Ruijs, M.D., Radboud University Nijmegen Medical Centre, Department of Public Health, 117 SG, PO Box 9101, 6500 HB Nijmegen, The Netherlands. (Email: h.ruijs@sg.umcn.nl)
}

children. Despite vaccination coverage of $>95 \%$, a rubella epidemic occurred in 2004/2005 and similar to rubella outbreaks among the Amish in the USA [2], this epidemic was largely confined to an orthodox Protestant minority group that refrains from vaccination for religious reasons [3].

To prevent CRS, all young women should be protected against rubella either via vaccination or the acquisition of a natural immunity prior to childbearing age. During the 2004/2005 epidemic, the Dutch municipal health services offered free MMR vaccination to unvaccinated children and adolescents. However, the acceptance of this was very limited. 
Similarly, a personal recall for missed vaccinations to unvaccinated 16-year-olds (as registered in the Provincial Vaccination Register) also showed only 7\% vaccination acceptance [4].

In contrast to the low vaccination acceptance rates, the unvaccinated rubella patients and their parents proved very willing to undergo diagnostic procedures to confirm rubella infection during the epidemic despite the results having no therapeutic consequences for them. Given the interest of unvaccinated girls and their parents in the serostatus of the girls, it was decided to develop a screening programme to detect rubella susceptibility among unvaccinated young women, offer MMR vaccination to those found to be seronegative, and thereby increase protection against rubella. This strategy has been suggested by others [5], and the objective of the present study was therefore to test the feasibility of such a strategy.

All 640 women aged 14-20 years from two villages with large unvaccinated orthodox Protestant populations were invited to take part in the study. Overall MMR vaccination coverage in these villages was $63 \%$ for the birth cohorts invited for the study. The target group of the programme was unvaccinated young women but to avoid stigmatization, the serological test was offered to all young women, irrespective of vaccination status.

Invitations for the serological test and questionnaires accompanied by an informed consent form were mailed to all of the women in the study population. For girls aged $<18$ years, the parents were approached and asked to provide written consent.

Vaccination status was assessed retrospectively via the questionnaire. Women who did not know their vaccination status were assumed to be unvaccinated.

Blood samples were taken in the villages by nurses from the municipal health service.To foster participation, the blood samples were collected via finger prick, which has been shown to allow sufficiently sensitive serological testing relative to testing of serum collected via venepuncture [6, 7]. Blood obtained via finger prick was spotted on filter paper and dried. The blood specimen was reconstituted in the laboratory and tested for the presence of rubella-specific IgG antibodies (Enzygnost ${ }^{\circledR}$, Dade Behring $\mathrm{GmbH}$, Germany). Rubella IgG test results $<4 \mathrm{IU} / \mathrm{ml}$ were classified as negative; test results $\geqslant 15 \mathrm{IU} / \mathrm{ml}$ were classified as protective; test results between 4 and $14 \mathrm{IU} / \mathrm{ml}$ were classified as equivocal. Women with initially equivocal test results were asked to provide a second blood sample but now via venous puncture.
Table 1. Characteristics of respondents

\begin{tabular}{lrr}
\hline \hline & $n$ & \multicolumn{1}{c}{$\%$} \\
\hline Age (years) & & \\
14-17 & 205 & $66 \cdot 8$ \\
$18-20$ & 102 & $33 \cdot 2$ \\
Total & 307 & $100 \cdot 0$ \\
Education & & \\
Low & 205 & $67 \cdot 0$ \\
High & 101 & $33 \cdot 0$ \\
Total & 306 & $100 \cdot 0$ \\
Religion & & \\
Orthodox Protestant & 87 & $28 \cdot 8$ \\
Other religions & 140 & $46 \cdot 4$ \\
No religion & 75 & $24 \cdot 8$ \\
Total & 302 & $100 \cdot 0$ \\
Vaccination status & & \\
Vaccinated & 199 & $64 \cdot 8$ \\
Not vaccinated & 91 & $29 \cdot 6$ \\
Unknown by respondent & 17 & $5 \cdot 5$ \\
Total & 307 & $100 \cdot 0$ \\
\hline \hline
\end{tabular}

These blood samples were again tested for rubella $\operatorname{IgG}$ using another test (AxSYM ${ }^{\circledR}$, Abbott Diagnostics, USA) due to the different logistics associated with the collection of the different blood samples. Venous blood test results $\geqslant 15 \mathrm{IU} / \mathrm{ml}$ were considered protective.

All of the participants received personal written feedback regarding the laboratory results. Unprotected women were offered MMR vaccination free of charge.

Only the analyses of the data from the subgroup of previously unvaccinated participants are presented here. Discussion of waning immunity among the subgroup of vaccinated women is beyond the scope of this report. The data were analysed using SPSS software, version 13 (SPSS Inc. USA). Percentages were calculated for participation, rubella susceptibility, and acceptance of vaccination. The different subgroups of participants classified according to religious denomination, age, and education were compared using Fisher's exact tests.

The participation in our study was $48 \%$ (95\% CI 44-52). A total of 307 women participated in the study of whom $108(35 \%, 95 \%$ CI $30-41)$ belonged to the target group of unvaccinated women. The characteristics of the participants are presented in Table 1. Vaccination status was significantly related to religious denomination. The majority of the unvaccinated women ( 77 women, $71 \%$ ) belonged to orthodox Protestant denominations. Vaccination status was not associated with age or educational level. 
Table 2. Rubella susceptibility of unvaccinated women according to religion and age $(n=108)$

\begin{tabular}{|c|c|c|}
\hline & $\begin{array}{l}\text { Percentage } \\
\text { susceptible }(n)\end{array}$ & $\begin{array}{l}\text { Percentage } \\
\text { protected }(n)\end{array}$ \\
\hline Total & $11 \%(12)$ & $89 \%(96)$ \\
\hline \multicolumn{3}{|c|}{ According to religion $* \dagger$} \\
\hline Orthodox protestant & $4 \%(3)$ & $96 \%(74)$ \\
\hline Other or no religion & $31 \%(9)$ & $69 \%(20)$ \\
\hline \multicolumn{3}{|l|}{ According to age } \\
\hline $14-17$ years & $4 \%(3)$ & $96 \%(67)$ \\
\hline $18-20$ years & $24 \%(9)$ & $76 \%(29)$ \\
\hline
\end{tabular}

* Information on religious denomination was missing for two respondents.

$\dagger$ Fisher's exact test: $P<0 \cdot 001$.

$\ddagger$ Fisher's exact test: $P=0 \cdot 004$.

Eleven per cent (95\% CI 6-19) of the unvaccinated women were susceptible to rubella. The results of the serological testing of the finger prick blood from 108 unvaccinated women showed five $(5 \%)$ to be seronegative, $10(9 \%)$ to have equivocal results and 93 $(86 \%)$ to be protected against rubella. After venous blood testing for those with initially equivocal results, three more women could be considered protected as their venous rubella $\mathrm{IgG}$ was $\geqslant 15 \mathrm{IU} / 1$; five women were unprotected, and two women did not return for repeated testing and were therefore also considered unprotected. Therefore, 12 (11\%, 95\% CI 6-19) unvaccinated women were considered susceptible to rubella. Rubella susceptibility in the unvaccinated women was clearly associated with religious denomination. A higher percentage of the women belonging to orthodox Protestant religious denominations were protected against rubella when compared to the women belonging to other religious denominations or women with no religious denomination (Table 2). In addition, a higher percentage of the younger unvaccinated women (i.e. those aged 14-17 years) were protected against rubella when compared to the older group (i.e. those aged 18-20 years). Rubella susceptibility in unvaccinated women was not associated with educational level.

Only $17 \% \quad(2 / 12,95 \%$ CI $2-48)$ of rubellasusceptible, formerly unvaccinated women agreed to subsequent MMR vaccination by the municipal health service. Both women did not belong to an orthodox Protestant denomination.

These outcomes were used to assess the feasibility of the screening and vaccination programme. The efficiency of the programme is dependent on the participation rate of the target group, their rubella susceptibility and the acceptance of vaccination by those susceptible. Thus it can be concluded that $0 \cdot 48 \times 0 \cdot 11 \times 0 \cdot 17 \times 100=0.9 \%(95 \%$ CI $0 \cdot 1-2 \cdot 5)$ of the target group of unvaccinated young women was provided protection by the programme. In other words: the invitation to 100 unvaccinated young women for rubella screening will lead to acceptance of a vaccination offer by only 1 susceptible woman. Ten women will remain susceptible because they do not agree to screening $(0 \cdot 52 \times 0 \cdot 11 \times 100=6$ women $)$ or refuse vaccination after testing seronegative $(0.48 \times$ $0 \cdot 11 \times 0 \cdot 83 \times 100=4$ women). Eighty-nine out of 100 unvaccinated women are already protected by naturally acquired immunity.

Our results show that rubella screening of unvaccinated women prior to childbearing age, followed by the offer of MMR vaccination to those who tested seronegative has only a very limited effect on rubella protection in an area with low vaccination coverage due to religious objections.

The participation rate in our study was $48 \%$. As the vaccination coverage found for our study population is consistent with the historical vaccination coverage, it could be assumed that the participation rate for the target group of unvaccinated young women was independent of their vaccination status and equal to the overall participation rate. Moreover, the participation rate found in the present study is comparable to the participation rate of $52.5 \%$ found in a 1996 population-based immunosurvey of low vaccine coverage municipalities in The Netherlands [8]. The necessity of parental consent for girls aged $<18$ years did not affect participation. The participation rate for the younger group was even higher than the participation rate for the older group. The aim of the screening and vaccination programme is to prevent rubella in pregnancy; therefore, the fact that for most women aged between 14 and 20 years, pregnancy is not yet an issue may contribute to the relatively low participation rates we found. Preconception rubella screening and vaccination may thus result in higher participation rates and has recently been recommended by the Dutch Health Council [9].

Rubella susceptibility among unvaccinated young women was found to be only $11 \%$, which reflects the high likelihood of naturually acquiring the rubella infection in these villages. Before the 2004/2005 epidemic there must also have been circulation of rubella virus in The Netherlands, as rubella seroprevalence in areas with low vaccination coverage in the 1996 population-based serosurvey for the same generation 
of unvaccinated youth - who were then aged 5-10 years - was already about $65 \%$ [10]. The higher seroprevalence in unvaccinated orthodox Protestant women relative to other groups of unvaccinated women is consistent with the observation that most cases in the 2004/2005 epidemic were found to occur in groups who refrained from vaccination for religious reasons [3]. Similarly, the finding that the 14- to 17 -year-old unvaccinated women were better protected against rubella than the 18- to 20-year-old women may reflect the fact that the younger age group had a higher probability of exposure during attendance at secondary school (often an orthodox Protestant school) than the older age group, which no longer attended school.

The acceptance of the vaccination offer by the previously unvaccinated seronegative women was limited. However, we were unable to assess the acceptance of the offer with much precision due to the small number of women identified as susceptible. Nevertheless, the reasons for low vaccination acceptance may be similar to the reasons mentioned for low participation. Religious objections may also give rise to a conflict of conscience on the part of unprotected young women in particular. Moreover, as pregnancy is not as yet an issue, the decision to accept vaccination can also be postponed. Therefore, it is possible that a vaccination offer following preconceptional screening will result in higher rates of vaccination acceptance.

Applying the Wilson-Jungner criteria for mass screening adopted by the WHO in 1968, we conclude that there is a serious health problem, a suitable test and an appropriate 'treatment' (i.e. vaccination). However, at the observed levels of participation, rubella susceptibility and vaccination acceptance, the screening of unvaccinated women prior to child bearing age is most probably not a cost-effective strategy in The Netherlands and therefore not recommended.

\section{ACKNOWLEDGEMENTS}

We thank Dr A. M. van Loon, University Medical Center Utrecht for rubella IgG testing on finger prick blood specimens. This study was financially supported by Fonds OGZ, a fund established by the Dutch government to stimulate public health research.

\section{DECLARATION OF INTEREST}

None.

\section{REFERENCES}

1. Banatvala JE, Brown DW. Rubella. Lancet 2004; 363: 1127-1137.

2. Briss PA, et al. Rubella among the Amish: resurgent disease in a highly susceptible community. Pediatric Infectious Disease Journal 1992; 11: 955-959.

3. Hahné SJM, et al. Rubella epidemic in the Netherlands, 2004/05: awareness of congenital rubella syndrome required. Nederlands Tijdschrift voor Geneeskunde 2005; 149: 1174-1178.

4. Aardoom HA, et al. Recall for missed vaccinations of sixteen year old youngsters, is it useful? Experiences with the reformed denomination in Dordrecht. Infectieziektenbulletin 2002; 13: 62-64.

5. Robinson JL, et al. Prevention of congenital rubella syndrome - what makes sense in 2006? Epidemiologic Reviews 2006; 28: 81-87.

6. Helfand RF, et al. Comparative detection of measles and rubella $\mathrm{IgM}$ and $\mathrm{IgG}$ derived from filter paper blood and serum samples. Journal of Medical Virology 2001; 65: 751-757.

7. Condorelli F, et al. Detection of immunoglobulin $G$ to measles virus, rubella virus, and mumps virus in serum samples and in microquantities of whole blood dried on filter paper. Journal of Virological Methods 1994; 49: 25-36.

8. de Melker HE, Conyn-van Spaendonck MA. Immunosurveillance and the evaluation of national immunization programmes: a population-based approach. Epidemiology and Infection 1998; 121: 637643.

9. Health Council of The Netherlands. Preconception care: a good beginning. The Hague, Health Council of the Netherlands, 2007, publication no. 2007/19.

10. Haas de HR, et al. Prevalence of antibodies against rubella virus in The Netherlands 9 years after changing from selective to mass vaccination. Epidemiology and Infection 1999; 123: 263-270. 